- 论坛・

\title{
路径依赖下的物种形成机制
}

\author{
李敏岗1,2\#, 王超 $1 \#^{*}$, 王瑞武 $1^{*}$
}

1. 西北工业大学生态环境学院, 西安 710072; 2. 西北工业大学数学与统计学院, 西安 710072

摘要: 生物科学几乎所有研究都需要物种概念作为基础, 生物多样性研究亦需要可操作的物种概念, 但现有物种概念存在不 同程度的人为因素或难操作性，对物种划分造成不利影响。本文引入“进化路径”这一概念，说明适合度景观时刻变化着，物 种在每个进化时间点上依据瞬时适合度选择下一时刻的进化状态，且总是沿着动态适合度景观中适合度增加的方向进化。基 于演化博弯的方法，以随机过程为例模拟物种的进化过程。进而提出路径依赖下的物种形成机制，并在此基础上给出可操作 的物种定义, 即: 针对基因、性状、生态过程等任一状态下两个群体内个体的多个变量做统计分析, 若群体之间同时在两个 或多个维度状态下呈现出的不连续性 $d$ 大于群体内变量呈现出的差异性 $\sigma_{k}$, 则拥有相应变量的个体属于不同物种。

关键词: 物种; 物种形成; 演化博弯; 随机过程; 路径依赖

李敏岗，王超，王瑞武 (2021) 路径依赖下的物种形成机制. 生物多样性, 29, 409-418. doi: 10.17520/biods.2020276.

Li ML, Wang C, Wang RW (2021) Path-dependent speciation in the process of evolution. Biodiversity Science, 29, 409-418. doi: 10.17520/biods.2020276.

\section{Path-dependent speciation in the process of evolution}

\author{
Minlan $\mathrm{Li}^{1,2 \#}$, Chao Wang ${ }^{1 \# *}$, Ruiwu Wang ${ }^{1 *}$ \\ 1 School of Ecology and Environment, Northwestern Polytechnical University, Xi'an 710072 \\ 2 School of Mathematics and Statistics, Northwestern Polytechnical University, Xi'an 710072
}

\section{ABSTRACT}

Aim: Almost all the research in biology relies on a species concept as the basis for biodiversity. However, all of the existing species definitions are imprinted with artificial factors or difficult to observe in practical applications, which brings negative impacts on the species classification. Here, we introduce an "evolutionary path" using a path integral to provide a rule for species classification. We aim to show the speciation process and define the species concept with a mathematical form.

Methods: In this species definition, we assumed that uncertain environmental changes and random drift in the population might simultaneously lead to a change in the fitness expectation. Therefore, a constant fitness expectation for any biological characteristic might not be reliable when considering how characteristics vary through time and space. We introduce the concept of "evolutionary path" which is formed by repeating a short-time transfer process. In this process, a species evolves to different states at different probabilities over time based on the instantaneous fitness landscape at any current moment. In this framework, evolution moves in the direction of increased fitness on the varying fitness landscape, and speciation will be of path dependence on the varying fitness landscape. Different individuals with the same or different biological characteristics (e.g. phenotype, genotype, etc.) will interact with another one at random, similar to the process of gambling among them. In a simulation, under the framework of evolutionary game theory, species differentiation will be similar to the evolution of the peaks on a mountain. Every peak after differentiation may represent a species, a cryptic species, or a sympatric species. The picture of species peaks within a mountain is determined by the relationship between the distance and the width of two adjacent peaks and by the dimensionality that characteristics differentiation satisfied.

Results: We found a more practicable concept to define species, i.e, based on statistical analysis applicable for multiple types of traits like genetics, morphological characteristics, or ecological process between two populations. Once the

收稿日期: 2020-07-11; 接受日期: 2021-01-04

基金项目: NSFC-云南联合基金(U1302267)、国家杰出青年科学基金(31325005)和西北工业大学研究生创新团队建设项目

\# 共同第一作者 Co-first authors

* 通讯作者 Co-authors for correspondence. E-mail: chaowang@nwpu.edu.cn; wangrw@nwpu.edu.cn 
respective discontinuities of two or more dimensional variables between populations are all greater than the difference of variables within the population, the individuals with corresponding variables belong to different species.

Conclusions: The path-dependent evolutionary mechanism in this model demonstrated that species can coexist with different probabilities when environmental pressures are limited. A new species, cryptic species, sympatric species may occur in a path-dependent evolution process. This model also showed that species survival in an ecosystem is not determined by its fitness directly, but dependent on the probability of its evolutionary path.

Key words: species; speciation; evolutionary game; stochastic process; path dependence

物种是生物有机体分类和进化的最基本单元 (卢宝荣和王哲, 2016), 正确划分物种对生物多样性 的保护至关重要。但因思考的角度不同和基于的进 化时间不同, 现有各个物种概念只能反映进化路上 物种的一个侧面(刘建全, 2016)。从单个方面认识物 种的方式如同 “盲人摸象”, 并不全面, 给物种分类 和生物多样性的保护工作造成了极大的困难。目前 已有接近30个(Wilkins, 2009)甚至更多(周长发和杨 光, 2001; 洪德元, 2016)关于物种的定义。其中最常 用的物种概念分别强调了生殖隔离、基因差别、进 化、系统发生或支序分类理论、生态以及外部形态 (洪德元, 2016)。

运用这些概念对物种进行划分时均有一定的 局限性: 要么必须进行实验来确认它们是否存在生 殖隔离, 而这一过程往往难以操作且不能适用于所 有生物; 要么过于抽象, 如生态学物种概念; 还有 的虽然较为客观但却因过于简单而无法操作, 如 Hedberg (1958)提到的形态学物种概念。形态学物种 概念认为: 当两个种群仅在一个性状上呈现完全不 连续变异时(常伴有其他性状上的部分不连续) 就区 分为亚种, 而当它们之间的不连续差异表现在两个 或多个独立性状上时就一律区分为不同的物种 (Hedberg, 1958)。这虽然明确了可以依据形态上的 差异来区分物种, 但依然没有回答表型在种内的连 续差异和在种间的不连续程度具体为多少时才是 不同物种这一问题, 因此不具备较强的可操作性。 基于这些物种概念现状, 洪德元(2016)提出了可操 作的形态-生物学物种概念, 即: “物种是由一个或 多个自然居群组成的生物类群, 种内呈现形态性状 的多态性和变异的连续性, 而种间则有两个或多个 独立的形态性状显现变异的间断或统计上的间 断”。使用该定义对形态性状进行统计聚类分析时, 展示了变异的连续性与间断性, 但是仍然难以对处 于中间状态或偏离聚合群的多数物种作出判断。因
此, 必须有一个可以量化的指标来对物种进行合理 划分。

为解决上述问题, 正确认识物种的形成过程并 在此基础上给出物种的定义是关键点。我们引入 “进化路径”这一概念, 认为物种进化依赖一定的路 径。假设选择压力有限, 物种在追求高收益的过程 中将沿着概率最大或者稍低的路径分化得到不同 的物种。需要明确的是, 物种一直处于分化路上, 分化到后期的“好种”在基因型、性状、生态过程等 状态上都分化完全, 但实际上大多数物种处于分化 的前期和中期, 只满足一种或两种状态的分化, 且 在达到分化后期之前又进行新一轮的分化(刘建全, 2016)。结合实际情况, 本文在给出物种定义时考虑 两点: 一是明确不同物种不能同时满足 “好种” 所需 满足的所有物种概念条件。各条件存在分化的先后 (刘建全, 2016), 因此在某时刻只需考虑至少两种状 态, 使其满足完全分化独立的要求。二是明确大多 数物种处于分化的前中期而非后期, 很难满足各状 态(基因特征、形态特征、生态过程等)的完全隔离。 因此需要对“完全隔离”这一条件加以放松, 设定出 更加符合实际中分化独立的状态隔离条件。为简化 叙述, 本文统一从形态(表型)的角度出发去考虑物 种进化这一问题, 从基因层面或者生态过程的角度 讨论物种进化其机制也是类似的。

物种的存在状况可以由其适合度刻画, 因此其 进化结果反映在适合度景观(fitness landscape)上。 适合度景观是由被研究群体的表型分布形成的。从 表型角度出发的进化路径是一段时间内个体表型 在进化过程中所经历的所有状态。与Frank (2011) 考虑的静态适合度景观不同, 我们认为适合度景观 随着种群结构的变化也在时刻改变着。经典适合度 景观类似爬山的过程: 如果山峰峰值位置确定, 爬 上峰顶的预期就确定; 而当山峰形态和峰值是不断 变化着的时候(从地质的尺度来看, 山峰形态和峰 
值确实是变化着的), 个体爬山的过程就没有确定 的预期值, 我们只能描述个体爬山过程某一瞬时情 况的预期值(王瑞武, 2021)。在适合度景观上的物种 进化同样如此。

物种进化受到选择压力的影响。选择压力指的 是外界自然环境和来自周围个体的压力, 在每个时 刻都有所不同。在一个群体中, 假设所有个体的初 始表型相同, 但演化过程中受到选择压力的作用可 能会根据其适合度随机保持当前表型或突变为其 他表型。每个表型的适合度与拥有该表型的个体在 群体中的数量相关, 每个表型都对应着一个适合度, 所有表型及其适合度组成一个适合度景观。不同表 型在不同时刻的适合度不同, 即每个时刻存在瞬时 适合度景观, 因此在一段时间内形成动态适合度景 观。表型在这样的景观上游走而产生多条可能的进 化路径, 每条路径对应一个概率值, 在一条路径上 一个时间点内两个表型之间的转移概率与其适合 度有关。显然, 选择压力一定时, 不只是最大概率 的路径被选择, 而是选择允许多条路径存在。沿着 这些进化路径在不同时刻可以看到相同或不同的 物种, 取决于不同表型之间的(不)连续性差异程度。

假设群体内个体的表型服从正态分布, 最优表 型可以看作是该群体的平均表型。自然选择将允许 多条进化路径存在, 因此一段时间内进化路径与进 化结果存在几种情形: (1)选择压力始终较大时, 群 体的初始平均表型沿概率最大的路径从一个表型 进化到其他表型; (2)当每个时刻选择压力有限时, 每个时刻有多个表型被选择, 其进化路径也不再只 是概率最大的路径; (3)当前 $n-1$ 时刻的选择压力有 限, 第 $\mathrm{n}$ 时刻的选择压力很大时, 此时初始表型沿 多条路径进化到另一个表型值, 即单态群体沿不同 路径进化得到另一个单态群体。在某个特定时间点 观测时, 若沿不同路径进化出的不同表型之间满足 一定的数量关系, 且至少另有一个其他生物学特征 也满足类似关系, 便可将它们对应的个体定义为不 同物种。进化路径反映了自然选择的方向, 突变、 漂变等因素会对路径的选择造成随机扰动。引入 “进化路径”这一概念, 便很容易理解某一表型进化 过程中分化出有不同适合度表型的现象, 也因此对 由表型分化导致的物种形成有了更清晰的认识。

本文将从物种形成机制的基本模式出发, 基于 选择压力有限时所有个体的表型沿多路径分化且
其进化过程是 “路径依赖”的, 以Moran过程为例模 拟给出个体表型进化路径的直观展示。在随机因素 和选择作用的影响下, 状态频率的演化路径对应为 群体表型的分化路径, 沿不同路径的演化可能会进 化得到不同表型。通过讨论物种在动态适合度景观 上的适应性演化，分析表型在 $t_{0}-t_{n}$ 时间段内从 $x_{0}$ 到 $x_{n}$ 的进化路径, 认识物种从分化到形成新物种这一 过程。在此基础上通过数学形式给出可量化的物种 概念, 期望更好地界定物种。

\section{路径依赖下的物种进化}

物种是进化着的。拉马克最先提出进化思想, 认为物种随时间演替逐渐发生变化, 新的物种不断 产生(李启剑和李越, 2010), 但并未给出物种演变机 制。达尔文提出“物竞天择, 适者生存”, 认为变异是 生物的核心特征。适应环境的变异在自然选择的作 用下得到保存, 不适应的则被淘汰。即使他并未在 《物种起源》中给出具体的物种定义, 但其进化与 选择的思想对人们认识物种产生了深刻影响。达尔 文认为物种是变化着的, 不同物种可以由同一物种 演化而来(Darwin, 1929)。

但是物种演化的机制和路径却并不清楚。实际 上, 物种在进化过程中受到自然选择的压力和周围 个体的影响, 在每个时刻通过突变的方式以不同概 率选择下一时刻的进化状态, 一段时间内物种基于 动态适合度景观产生多条进化路径。物种依赖一定 的路径产生或灭绝, 体现了生命的连续性; 但从某 一时空瞬间对物种演化进行横向观测, 则会发现物 种的间断性，体现在表型、基因型、生态过程等状 态的不连续差异。那么, 物种出现这种进化结果的 原则是什么? 以表型为例, 实际上是拥有不同表型 的个体在自然选择下追求较高的收益, 整个进化过 程即为表型在适合度景观上的游走。因此, 下面首 先对适合度景观作以说明。

\section{1 适合度景观悖论}

已有研究大都建立在静态适合度景观的基础 上(Maynard Smith, 1982; Frank, 2011), 认为在一段 进化时间内适合度景观是确定的。进化可以看作是 一个生物体在有高峰和低谷的适合度景观上的旅 程。个体总是能够感受到适合度景观山脉上的峰值, 它试图向山上攀爬并努力达到局部或者全局的最 高峰, 适应意味着表型能够找到适合度景观的峰值 
并停留在那里(Nowak, 2006)。自然选择沿着适合度 增加的表型方向进化, 因此在有着多峰的适合度景 观(图1)中, 表型很容易被困在某一更低的局部峰值 上。Frank (2011)提出 “反应规范” 的概念来解决这一 困境。反应规范指的是拥有某表型的基因型对应的 (由发育等因素形成的)表型分布。单个基因型可以 对应多个表型值, 表型发育以适应不同的环境, 但 由于内在的发育波动以及外在的环境扰动使得表 型空间得以扩展。在一个有着多峰多谷的适合度景 观中, 发育变异性可以通过平滑函数将该景观处理 为有单峰的适合度景观, 这样可以加快表型的进化 速率。

但问题在于，实际上适合度景观并不是在每个 时刻都保持一致。表型受到环境的影响在适合度景 观上游走的过程中也同时影响适合度景观，因此每 个时刻存在一个瞬时适合度景观, 表型在每一个进 化时间点上处于与上一时刻不同的适合度景观中。 在静态适合度景观中每个时刻个体依据博弯矩阵 选择的所谓最优表型(收益最大)并不能在长时间后 使得该条进化路径被选择的概率最高。这一点可以 参考重复囚徒困境中TFT (tit-for-tat) 和ALLD (all defection)策略的博亦情况。TFT策略又称“以牙还

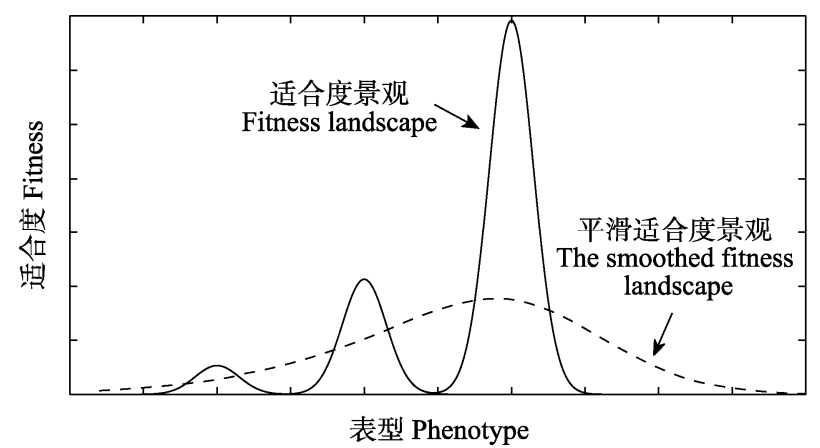

图1 表型的适合度景观图。实线表示真实的适合度景观, 当生物体在这样有高峰有山谷的适合度景观上进化时, 很 容易被困在某一更低的局部峰值上。虚线是在Frank提出的 反应规范作用下的表型适合度景观, 即由某基因控制的表 型的适合度景观, 该景观只有一个单峰, 使得表型更容易进 化到适合度更高的表型。

Fig. 1 Fitness landscape of phenotypes. The solid line shows the fitness landscape observed. When an organism evolves on such a multipeak fitness landscape, it's easy to be trapped at a lower local peak. The dashed line displays the phenotypic fitness landscape smoothed by reaction norm, i.e., the expect fitness for a genotype with a certain average phenotype. This fitness landscape just has one single peak, which makes it easier for any phenotype to evolve to another one with higher fitness.
牙”策略，是指在第一回合选择合作，而后每一回 合采用对手在上一回合中使用的策略; ALLD又称 “永远背叛”策略。在单次囚徒困境博弯中, 背叛永 远是最优策略。但是在多次博亦中, 当博亦次数大 于某一值时，TFT策略能够抵制ALLD策略的入侵 (Nowak, 2006)。

\section{2 进化路径}

基于静态适合度景观, Frank (2011)认为表型沿 最短的路径快速进化到高适合度表型; Traulsen等 (2007)认为表型沿时间最短的路径进化。而当表型 不再沿着固定适合度景观的山脊进化追求此种情 况下的最大适合度时, 表型进化应该选择怎样的路 径呢? 我们认为当选择压力始终很大时, 表型在进 化过程中几乎会一直沿着某条路径, 该路径是概率 最大的路径。然而, 实际上选择压力是有限的。一 个很自然的情形是, 群体内的表型在进化的过程中 产生多条进化路径, 此时自然选择不再只是倾向于 概率最大的路径, 其他概率稍低的路径也会被选 择。表型沿着这些路径进化得到可以被用来区分物 种的多个表型。为了明确“进化路径”这一概念, 我 们需要定义进化路径并观察表型在进化过程中所 有可能的路径分布。在每个时刻选择压力都是有限 的情形下，概率最高的一条路径会被大部分个体在 进化过程中所选择。其他概率较高的路径也会被选 择使得该表型进化到其他表型。另一种情形是，前 $n-1$ 时刻的选择压力有限，第 $n$ 时刻的选择压力无 穷，此时沿多条路径进化到一个表型(图2)。

我们探究 $t_{1}-t_{2}$ 时间段内表型从 $x_{0}$ 进化到 $x_{n}$ 的 进化路径, 个体在每一时刻都会根据其瞬时适合度 以一定概率去选择自己在下一时刻应该进化到的 表型状态, 因此表型沿着一定的路径进化。考虑进 化过程中 $n$ 个离散时间区间，则每个时刻对应表型 的出现概率为 $P\left[x_{i}\left(t_{i}\right)\right], x_{i}$ 表示该时刻的表型。受 随机漂变和突变的影响, $x_{i}$ 不止一种。假设路径 $a$ 上的表型为 $x_{0}, x_{1}, x_{2}, \ldots, x_{n}$, 则该路径出现的概率 为 $P\left[x_{0}\left(t_{0}\right), x_{1}\left(t_{1}\right), x_{2}\left(t_{2}\right), \ldots, x_{n}\left(t_{n}\right)\right]$, 也记为 $P[x(t)]$ 。 假设此概率是所有路径中概率最大的路径, 则当选 择压力很大时, 只有路径 $a$ 被选择; 而当选择概率 有限时, 路径 $b$ 也会被选择。至此, 表型进化路径的 说明已阐述清楚。 


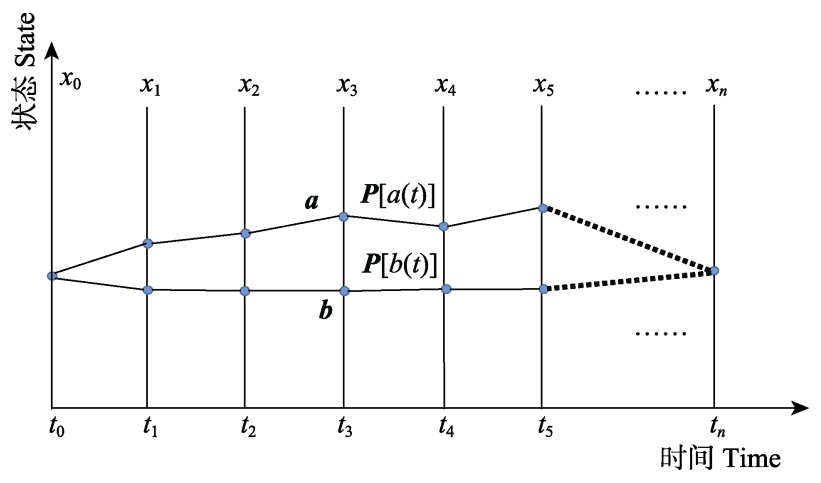

图2 群体内表型从 $x_{0}$ 进化到 $x_{n}$ 过程的示意图。个体在每 一时刻都会根据瞬时适合度以一定概率去选择自己下一时 刻期望进化到的表型, 因此群体内的表型从 $t_{0}$ 到 $t_{n}$ 的进化过 程中可能产生多条路径, $\boldsymbol{a}, \boldsymbol{b}$ 即为其中的两条。假设路径 $\boldsymbol{a}$ 上 的表型为 $x_{0}, x_{1}, x_{2}, \ldots, x_{n}$, 该路径出现的概率为 $P\left[\left(t_{0}\right), x_{1}\left(t_{1}\right), x_{2}\left(t_{2}\right), \ldots, x_{n}\left(t_{n}\right)\right]$ 。

Fig. 2 An evolutionary process for phenotype in a population from $x_{0}$ to $x_{n}$. An individual selects the next phenotype to evolve with a certain probability according to its instantaneous fitness at each time step, thus many possible paths of phenotype in a population occur including paths $a$ and $b$. Suppose that trajectory $a$ consists of phenotype $x_{0}, x_{1}, x_{2}, \ldots, x_{n}$, then the possibility is $P\left[x_{0}\left(t_{0}\right), x_{1}\left(t_{1}\right), x_{2}\left(t_{2}\right), \ldots, x_{n}\left(t_{n}\right)\right]$ 。

\section{2 基于路径的物种进化机制}

物种定义中的状态可以指基因、性状，或者生 态过程, 为方便理解物种定义的确定过程和路径依 赖下的物种形成过程, 下面将继续使用性状(表型) 这一状态加以叙述。在有限的选择压力下, 群体内 的表型从 $x_{0}$ 进化到 $x_{n}$ 有多条可能的路径。在所有个 体的表型都为 $x_{0}$ 的群体中, 个体在每个时刻依据 其瞬时适合度以一定概率选择下一时刻的表型状 态, 进化到每一个状态的概率理论上是不同的。个 体的瞬时适合度依赖于其表型在群体中的频率分 布, 此时演化博亦论就成为从表型这个角度思考生 物演化问题的一种方法(Maynard Smith, 1982)。

不失一般性, 我们以最简单囚徒困境为例利用 Moran过程说明个体表型的进化过程。在Moran过程 中, 某一个体有策略 $\mathrm{A}$ 和策略 $\mathrm{B}$ 。在有限群体中, 随机漂变和频率制约选择的交互作用决定了进化 博弯的结果。假定在一个大小为 $\mathrm{N}$ 的均匀混合的有 限群体中, 任意拥有不同表型的两个体之间进行博 弯, 其支付矩阵如下:

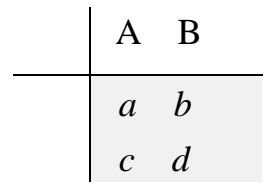

如果有 $i$ 个个体采取 A 策略, $N-i$ 个个体采 取 $\mathrm{B}$ 策略, 则采取 $\mathrm{A}$ 策略的个体平均收益为: $f_{i}=\frac{a(i-1)+b(N-i)}{N-1}$, 采取 B 策略的个体期望收 益为: $g_{i}=\frac{i c+d(N-i-1)}{N-1}$ 。在每一时间步,一个个 体以正比于其适合度的概率被随机选择并进行繁 殖, 同时在群体中随机挑选一个个体死亡, 前者后 代代替后者, 保证群体总体数量保持不变。因此, 在每个时间步时, A 的频率 $i$ 最多改变一个: $i$ 至 $i+1, i$ 至 $i-1, i$ 至 $i$, 其转移概率分别为:

$$
\begin{aligned}
P_{i, i+1}= & \frac{i f_{i}}{i f_{i}+(N-i) g_{i}} \frac{N-i}{N} \\
P_{i, i-1} & =\frac{(N-i) g_{i}}{i f_{i}+(N-i) g_{i}} \frac{i}{N} \\
P_{i, i} & =1-P_{i, i+1}-P_{i, i-1}
\end{aligned}
$$

对应于群体内有有限个离散策略的表型空间, 该模型中的频率状态也可指代具体表型, 此时频率 的转移概率即为两个离散表型之间的转移概率。只 是这样的转化规则尚不清楚, 因此仅以该模型的模 拟结果作为表型进化的示意图，二者演化原理类似。

通过模拟, 我们得到群体内表型进化过程的示 意图(图3，4)。图3显示了随着时间的演化个体表型 在进化过程中形成的不同路径, 即表型的进化路径 空间。沿着概率较高的几条路径进化便会产生新物 种。图4描述了表型沿路径连续演化的频率分布图。 可以看到, 群体内初始表型值为50, 其频率分布曲 线是单峰, 最高频率为 $90 \%$, 意味着群体内几乎所 有个体都拥有同样的表型。随着时间的演化, 初始 表型逐渐沿特定路径分化, 在 $t=600$ 时出现明显的 分化, 其频率分布曲线显示为双峰分布, 意味着群 体内个体的表型出现了分化, 此时群体内的表型均 值分别为 25 和 28 , 可以结合其他的生物学特征进一 步判断相应的个体是否为不同物种。不同时刻的频 率分布曲线分别显示为类似于一维或多维正态分 布的曲线，不同表型均值体现了种间表型的不连续 差异, 方差体现了种内表型的连续差异。其他状态 量在进化过程中也遵循同样的机制。 


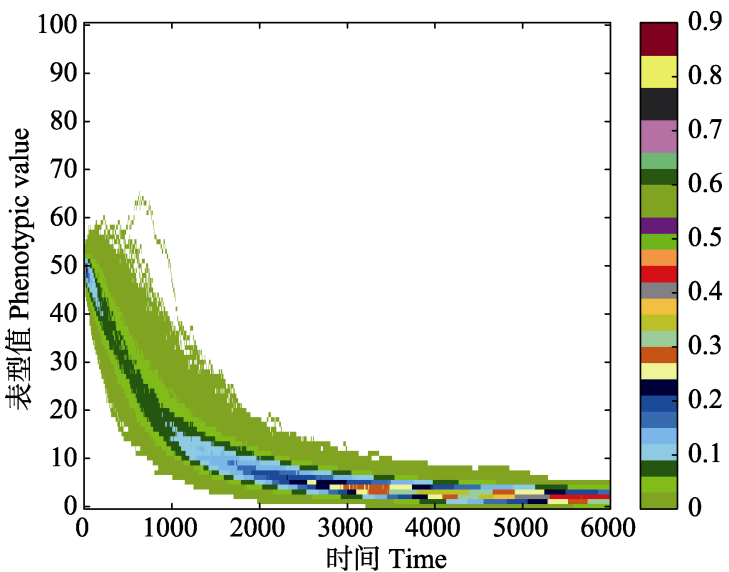

图3 进化路径的分布。不同颜色表示路径的频率。随着时 间的演化, 表型从最初的状态分化出其他表型, 在此过程中, 表型只能向相邻的表型进化, 因此进化是连续的。若在 $t=$ 1,000 这一时刻观察演化结果, 则可以看到明显不连续的物 种分化(两个蓝色区域)。

Fig. 3 The distribution of evolutionary paths. Different colors represent different distribution probability of paths. As time goes by, one phenotype differentiates to other phenotypes with different probabilities. The evolutionary path is continuous in this process because that phenotypes can only evolve to adjacent phenotypes. If the evolutionary results are observed at the moment of $t=1,000$, significant discontinuous phenotype differentiation (blue regions) can be seen.

当所有个体的表型沿不同路径进化到不同表 型状态时, 出现多表型共存的情形, 同时存在洪德 元(2016)定义的形态-生物学概念中所提到的种内 呈现的形态性状的多态性和变异的连续性。我们关 注分布密度较高的表型, 若它们之间满足统计数量 关系(体现在密度差异较小, 表型差异性较大), 则 可以认为这样的表型是分化成功的表型, 可以作为 物种判定的依据。

任意时刻在自然选择的作用下都存在群体内 表型的分布(如图5a-c)。以表型 $x_{1}$ 的进化过程为例, 图5a展示了该表型分化初期某时刻的分布, 当该表 型在之后的某一时刻出现图5b所示的分布, 则表型 $x_{1}$ 分化成两个表型 $x_{11}$ 和 $x_{12}$ 。方便起见, 同时考虑 对应物种的另一状态量一一基因型 $x_{2}$ 。在同样的演 化机制下, 群体内的基因型 $x_{2}$ 分化成两组不同基 因型 $x_{21}$ 和 $x_{22}$ (图5c)。此时若两群体的个体同时在表 型和遗传层面拥有分化后的特征则可进一步判断 它们是否为同一物种。显然, 除了表型和遗传层面, 在其他任意至少两个状态量上表现出类似的分化 过程, 均可作为物种判定的条件。
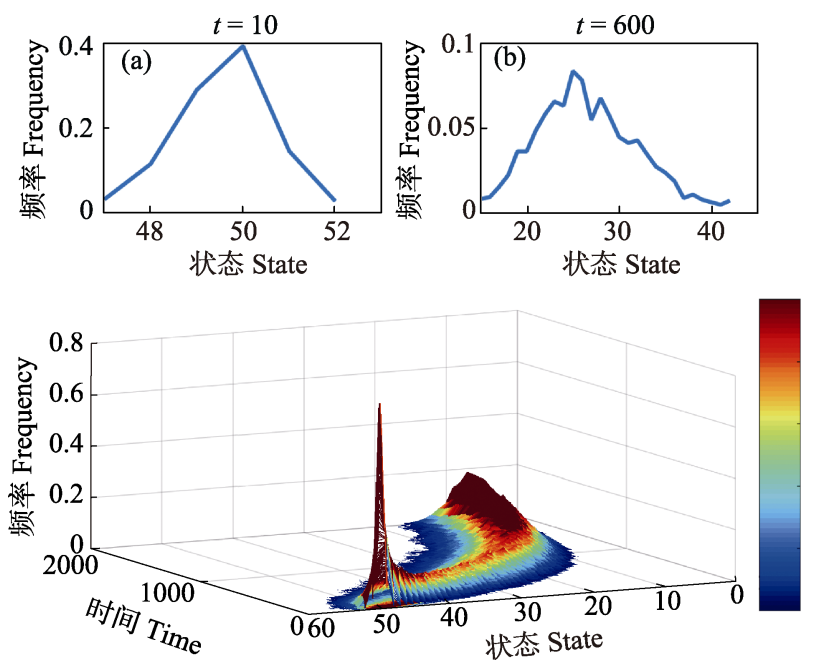

图4 路径依赖下的状态进化。(a)状态量随时间演化的频率 分布。状态量可以指基因型、性状特征、生态过程。(b) $t=10$ 时的表型频率分布。(c) $t=600$ 时的表型频率分布。若以表 型作为状态量, 分化初期显示为单峰的频率分布, 在时间 $\boldsymbol{t}$ $=600$ 处时出现明显的分化且显示为双峰的频率分布。这意 味着群体内个体从初始的单一表型(50)逐渐分化成两种表 型(25和28), 可以进一步判断对应的个体是否为不同物种。

Fig. 4 Path-dependent evolutionary process of states. (a) The frequency distribution curve of states over time. The states represent genotype, phenotype or ecological process. (b) The frequency distribution curve of phenotypes at $t=10$, (c) The frequency distribution curve of phenotypes at $t=600$. From the perspective of phenotype, the distribution curve changes from unimodal in the early phase to bimodal at $t=600$, which means the phenotype differentiates into two phenotypes (25 and 28) at $t=600$ from one phenotype (50) in the initial stage. Then we can judge if the species with phenotype 25 and 28 are different species.

表型等状态量出现明显分化是新物种的形成 条件之一, 一个直观的问题是: 分化界限是什么? 即分化程度为多少时, 可以作为新物种形成的判断 条件? 下面我们将对此展开讨论。

\section{物种的界定}

\section{1 路径依赖下的物种形成过程}

基于对路径依赖下物种形成机制的理解，我们 给出物种定义的数学形式, 从而为判断两相似物种 是否为同一物种提供量化指标。对于一个处于分化 路上的物种 $\mathrm{A}$, 考虑其两个状态量 $x_{1}, x_{2}$, 那么在 不同进化时间物种的存在状况亦有所不同(图6)。

依据观测时间 $t$ 的不同, 物种存在以下情形:

$$
\left\{\begin{array}{l}
\text { 一个物种, } t \leq t_{1} \\
\text { 一个物种, } t_{1}<t \leq t_{2} \\
\text { 两个物种, } t>t_{2}
\end{array}\right.
$$




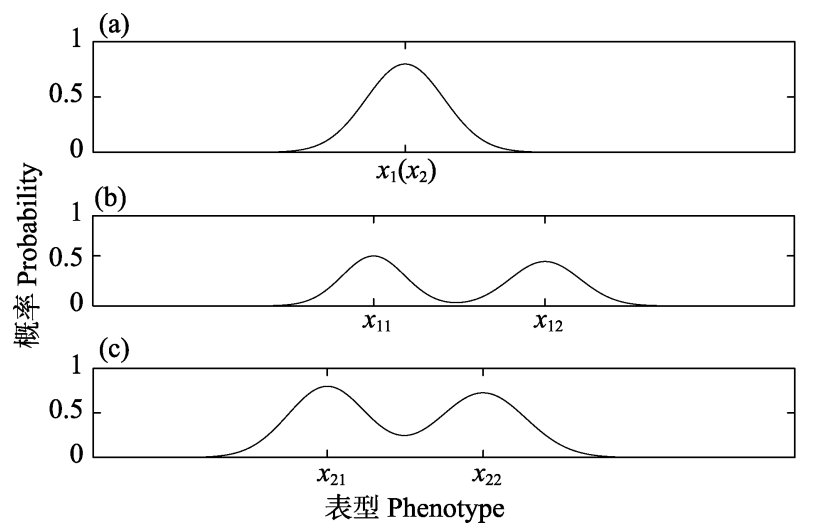

图5 不同时刻下表型和基因型的频率分布。(a)表示状态量 $x_{1}\left(x_{2}\right)$ 未分化初期某时刻的频率分布; (b)表示群体内的表 型 $x_{1}$ 在分化之后某时刻的频率分布; (c)表示群体内的基因 型 $x_{2}$ 在分化之后相同时刻的频率分布。假设对于原始物种 A, 其任意在两个状态 $x_{1}$ 和 $x_{2}$ 随着时间的演化受到基因突 变和随机漂变的影响, 在相同或不同时刻出现不同程度的 分化。当我们处于物种进化的一个“切面”去观察时, 表型 $x_{1}$ 分化成 $x_{11}$ 和 $x_{12}$, 并且进化依赖于特定的路径。若此时分化 出的表型和基因型在数量统计上满足一定的条件, 同时在 表型和基因型两个状态下同时具有特征 $x_{11}, x_{21}\left(x_{11}, x_{22}\right.$ 或 $x_{12}, x_{21}$ 或 $x_{12}, x_{22}$ ) 的个体为新物种。

Fig. 5 Frequency distribution of phenotypes and genotypes at different time. (a) showing the frequency distribution curve of the state $x_{1}\left(x_{2}\right)$ in the early differentiation. (b) demonstrating the frequency distribution curve of the phenotype $x_{1}$ at a certain moment after differentiation. (c) indicating the frequency distribution curve of the genotype $x_{2}$ at the same moment after differentiation. Suppose that any two states $x_{1}$ and $x_{2}$ of the original species A evolved influenced by random mutation and drift, then different degree of differentiation occurs in the same or different time. When observing in a "cutting plane" of a species evolution, we notice that $x_{1}\left(x_{2}\right)$ differentiates into $x_{11}\left(x_{21}\right)$ and $x_{12}\left(x_{22}\right)$, and the evolution are path-dependent. Meanwhile, if the differentiated phenotypes and the genotypes in a quantity statistics satisfy certain conditions, the individuals with $x_{11}, x_{21}\left(x_{11}, x_{22}, O x_{12}, x_{21}, O x_{12}, x_{22}\right)$ are new species, respectively.

物种一直处于分化路上，完全分化独立的“好 种”满足充分的生殖隔离、形态隔离、生态位隔离 等现有不同物种概念所强调的不同侧面的条件(刘 建全，2016)。但实际上完全充分的隔离鲜有存在， 并且绝大多数物种处于分化的早期和中期, 在尚未 达到物种分化的最后期时便又进行新一轮的分化, 只满足部分物种概念(刘建全, 2016)。因此, 一方面 不能要求物种判定的条件满足所有物种概念, 另一 方面也需要考虑对隔离条件稍加放松, 比如将条件 改为“种间生殖隔离程度远大于种内群体间生殖隔

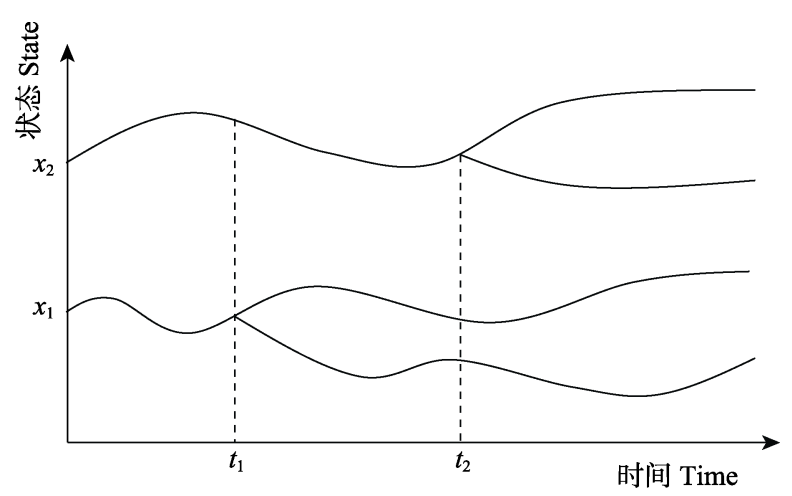

图6 随时间演替的物种界定示意图。状态量 $x_{1}\left(x_{2}\right)$ 在 $\boldsymbol{t}_{\mathbf{1}}\left(\boldsymbol{t}_{\mathbf{2}}\right)$ 时刻出现分化, 在不同的时间点上根据分化情况判定物种 是否属于新物种。

Fig. 6 schematic diagram of the species delimitation over time. The state $x_{1}\left(x_{2}\right)$ differentiates at $t_{1}\left(t_{2}\right)$. It can be determined whether a specie at different moments is a new species.

离程度” (刘建全, 2016)、“种间形态隔离程度远大于 种内群体间形态隔离程度”。那么, 从形态学角度出 发, 这些种内和种间的差异应满足什么条件? 这里 给出数学形式上的直观显示。需要说明的是, 在分 析基因或者生态过程等其他生物学特征时，只需满 足类似于表型差异中提到的关系即可。

以一个物种的表型状态量 $x_{0}$ 为例, 当进化沿 着特定的路径达到 $t$ 时刻时, 表型的适合度景观为 $f(x)$, 假设:

$$
f(x)=\sum_{i=1}^{n}\left(3\left|1+x_{i}\right|^{2}+1\right) N\left(x_{i}, \sigma^{2}\right)
$$

在当前进化时间点处, 适合度景观稳定，那么 使得 $f(x)=0$ 的平衡点 $x_{i}$ 即为此时群体内表型 $x_{0}$ 的进化结果, 记平衡点的个数为 $n$, 则若 $n>1$, 说 明表型分化。同时记分化后任意两个均值表型之间 的距离为 $d$, 那么当分化后的表型满足公式:

$$
\frac{d}{\sigma_{k}}>1(k=i, j)
$$

其中 $d=\left|x_{i}-x_{j}\right|$, 便可认为表型分化达到了形态 学物种概念中强调的形态上的隔离条件(图7)。这里, $\sigma_{k}$ 衡量了种内均值为 $x_{k}$ 的表型在形态上的连续变 异范围, $d$ 衡量了种间任意两个均值表型之间的不 连续差异, 公式(6)满足形态学概念下的物种判定条 件: 种间形态隔离程度远大于种内群体间形态隔离 程度。 


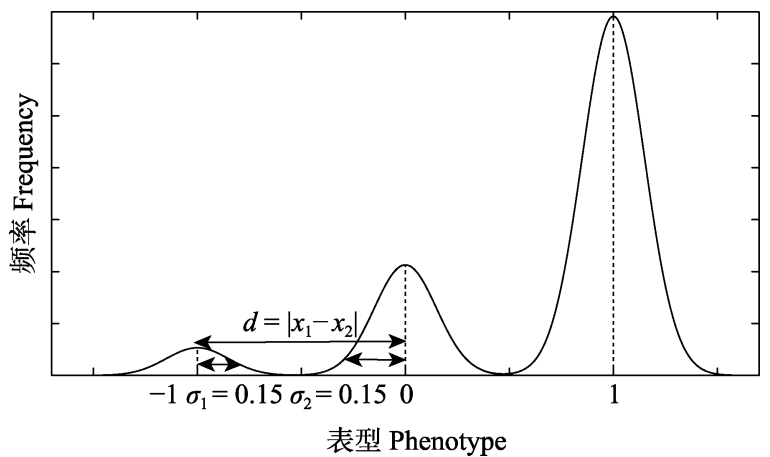

图7一表型在某一时刻分化出三个独立表型的示意图。该 图作为判定表型分化成功的一个特例, 此时种内均值为 -1 的表型存在连续且程度较低的差异(差异范围是士 0.15), 均 值为 0 的亦然。而这两个种间表型的不连续性达到了 1 , 其不 连续差异程度远高于种内连续差异程度, 因此可以认为该 表型达到了形态学物种概念中对于形态的要求。同时考虑原 始物种的其他状态上的分化, 若满足同样条件的差异, 即可 将相应个体确定为新物种。

Fig. 7 Differentiation of three independent phenotypes from one phenotype of a species at a moment. The figure is a special case of judging whether the phenotypic differentiates completely. There is a continuous and a little difference for the phenotype -1 within the species (the difference range is \pm 0.15 ), and the phenotype 0 versa. The discontinuity of these two interspecies phenotypes is 1 , and the discontinuity is much higher than the intraspecies continuous difference. Therefore, it can be considered that the phenotype meets the requirements for phenotype in the concept of morphological species. Taking into account the differentiation of another state of this species, if the difference satisfies the same conditions, the corresponding individuals can be determined as a new species.

\section{2 物种的定义}

针对基因、性状、生态过程等任一状态下两个 群体内个体的多个变量做统计分析, 若群体之间同 时在两个或多个维度状态下呈现出的不连续性 $d$ 与 群体内变量呈现出的差异性 $\sigma_{k}$ 满足 $\frac{d}{\sigma_{k}}>1(k=i, j)$, 其中 $d=\left|x_{i}-x_{j}\right|$, 则拥有相应变 量的个体属于不同物种。

\section{3 物种的判定}

事实上, 基因型、表型以及物种都是在各自层 面的适合度景观上游走, 因此上述界定物种的形式 可以抽象为一个共同范式, 应用于这三个方面。下 面我们仍然从形态这一角度出发, 利用上述定义判 断一些更一般的情形: (1)种间形态的不连续差异明 显大于种内形态的连续差异(图8a); (2)种间形态的 不连续差异不大于种内形态的连续差异(等于其中 一个种内的连续差异) (图8b); (3)种间形态的不连 续差异明显小于种内形态的连续差异(图8c); 在图 8(a)中, 两个相似群体间的表型均值之间的距离是 $d=\left|x_{1}-x_{2}\right|=|(-6)-6|=12$, 而群体内表型差异分 别是 $\sigma_{1}=3.0, \sigma_{2}=3.5$, 显然有 $12>3.0$ 且 $12>3.5$, 满足公式(6)所定义的物种定义, 因此若另有一生物 学特征也符合上述定义, 那么对应的个体属于不同 的物种。在图 $8 \mathrm{~b} 中$, 我们考虑一种看起来似乎难以 判断的情形, 图中这两个相似物种是同一物种吗?
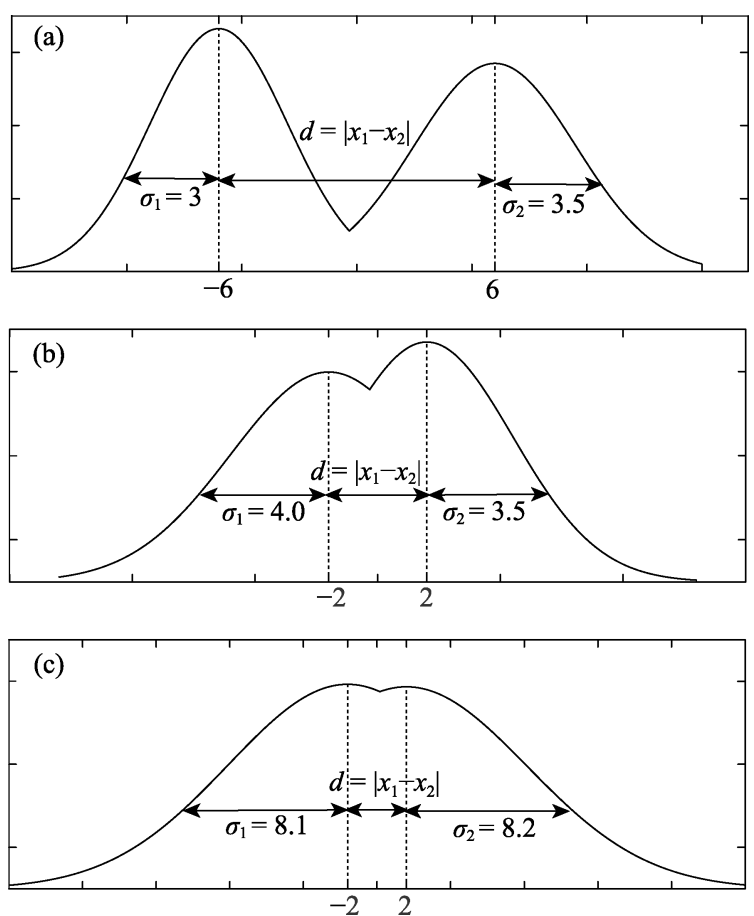

图8 不同的表型分化情形。如果已有一个生物学特征满足 定义中的差异性关系, 再考虑性状这一特征。图(a)中, $d=\left|x_{1}-x_{2}\right|=|(-6)-6|=12, \sigma_{1}=3, \sigma_{2}=3.5, d>\sigma_{1}$ 且 $\boldsymbol{d}>\sigma_{2}$, 若有表型分化满足此条件, 则对应个体属于不同物种; 图(b) 中, $d=\left|x_{1}-x_{2}\right|=|(-2)-2|=4, \sigma_{1}=4, \sigma_{2}=3.5, d=\sigma_{1}$ 且 $\boldsymbol{d}>\boldsymbol{\sigma}_{2}$, 若有表型分化满足此条件, 则对应个体属于同一物 种; 图 (c) 中, $d=\left|x_{1}-x_{2}\right|=|(-2)-2|=4, \sigma_{1}=8.1, \sigma_{2}=8.2$, $d<\sigma_{1}$ 且 $d<\sigma_{2}$, 若有表型分化满足此条件, 则对应个体属 于同一物种。

Fig. 8 Different case of phenotype differentiation. Imagine the relation of difference has been satisfied for one biological character. Then we consider phenotypes. In figure 8(a), $\quad d=\left|x_{1}-x_{2}\right|=|(-6)-6|=12, \sigma_{1}=3, \sigma_{2}=3.5, d>\sigma_{1}$ and $d>\sigma_{2}$. If individuals in two populations satisfy these conditions, they are different species. In figure 8(b), $d=\left|x_{1}-x_{2}\right|=|(-2)-2|=4, \sigma_{1}=4, \sigma_{2}=3.5, d=\sigma_{1} \quad$ and $d>\sigma_{2}$. If those individuals satisfy these conditions, they are the same species. In figure 8(c), $d=\left|x_{1}-x_{2}\right|=|(-2)-2|=4, \sigma_{1}=8.1, \sigma_{2}=8.2, d<\sigma_{1} \quad$ and $d<\sigma_{2}$. If those individuals satisfy these conditions, they are the same species. 
按照公式(6)的判断方法, 容易得到它们属于同一物 种的结论。考虑其中一个状态量有差异的物种, 可 以看到表型均值之间的距离是 $d=\left|x_{1}-x_{2}\right|=|(-2)-2|=4$, 而两个表型均值的连 续差异分别为 $\sigma_{1}=4.0, \sigma_{2}=3.5$, 出现 $d=\sigma_{1}$ 的情 形, 因此即使有另一生物学特征的分化满足公式(6) 条件, 这些表型对应的个体依然属于同一物种。在 图8c中, 考虑物种看起来极为相似的情形, 两个相 似群体间的表型均值之间的距离是 $d=\left|x_{1}-x_{2}\right|=|(-2)-2|=4$, 而群体内部表型差异 分别是 $\sigma_{1}=8.1, \sigma_{1}=8.2$, 显然 $4<8.1$ 且 $4<8.2$, 不满足公式(6)关于物种的定义。因此同样地, 即使 有另一生物学特征的分化满足公式(6)条件, 该表型 对应的个体依然属于同一物种。

\section{4 结论与讨论}

现有的物种概念仅反映了进化物种在分化至 后期需满足的不同条件之一, 因此不够全面。同时, 大多数物种概念具有主观性和难操作性, 因而更加 客观的物种概念亟待提出。基于这样的现状，本文 引入 “进化是路径依赖的” 这一思路, 以Moran过程 为例进行模拟，展示并说明了路径依赖下的物种形 成过程, 并在此基础上给出了可量化的物种概念: 针对基因、性状、生态过程等任一状态下两个群体 内个体的多个变量做统计分析, 若群体之间同时在 两个或多个维度下呈现出的不连续性 $d$ 与群体内变 量呈现出的差异性 $\sigma_{k}$ 满足: $\frac{d}{\sigma_{k}}>1(k=i, j)$, 其中 $d=\left|x_{i}-x_{j}\right|$, 则相应个体属于不同物种。

物种的定义和分类是生物学家一直以来关注 的重点。达尔文认为现有物种是由别的物种进化而 来的, 结合Hedberg (1958)和洪德元(2016)关于物种 的定义, 我们给出上述物种定义。其优势一方面在 于它的客观性, 另一方面在于它在统计表型差异时 的独立性。对于不同群体的任意表型，仅考虑它们 之间的相对差异, 而不是对所有物种的不同表型采 用同一套差异程度的衡量标准，造成划分的不准确 性。另外有几点需要说明:

(1)本文虽然自始至终围绕表型的进化过程进 行讨论, 但实际上基因型和生态过程的分化过程也 遵循类似的机制, 在物种判定时也满足一致的差异
关系。因为物种一直处于分化路上，对于来自同一 个最近共同祖先的两个分化至后期的“好种”来说， 既有形态上的差异, 又有程度较高的生殖隔离, 还 有明显的生态过程分化(刘建全, 2016)。

(2)从路径依赖的角度看, 本文只是强调选择 压力有限时物种是依赖较高概率的路径形成的, 这 样的路径是个体在进化过程中于动态适合度景观 中追求高收益产生的。而对于某一种内出现的个别 有别于种内其他个体、近似于另一物种的个体，从 生物学角度看是出现了基因或者表型回流的情形。 正如刘建全认为的: 物种是处于分化路上的，在还 未达到分化后期即尚未满足所有物种概念时，又会 进行新一轮的分化，此时种间出现基因流，产生了 杂种(刘建全, 2016)。实际上, 这些杂交个体的进化 也是依赖于各自的路径: 当其路径与选择不匹配时, 不能形成新的物种; 但是, 当杂交个体的遗传组 合、遗传新变异和分布地理区域的自然选择相吻合 时，则可能形成新的杂交物种(Abbott et al, 2013)。

(3)物种的分化时间与物种的形成无必然联系 (刘建全, 2016), 越早分化成种可能性越大。物种只 有某一表型最先开始分化时, 形成的可能只是变种; 但是, 变种和种在本质上没有区别(Darwin, 1929)。 特定类群物种界定操作过程中, 形态、生殖隔离、 生态等多方面群体水平上统计学差异的“整合物种 概念”仍具有一定的实践意义(刘建全，2016)。虽然 本文给出了物种分化路上的数学动态过程和一个 可量化的数学物种定义, 但其间仍有一些问题尚待 进一步探究, 比如: 在表型进化的过程中, 从一个 表型进化到另一个表型的进化概率应该如何确定? 这或许需要以数学和物理学为工具进一步研究。

\section{参考文献}

Abbott R, Albach D, Ansell S, Arntzen JW, Baird SJE, Bierne N, Boughman J, Brelsford A, Buerkle CA, Buggs R, Butlin RK, Dieckmann U, Eroukhmanoff F, Grill A, Cahan SH, Hermansen JS, Hewitt G, Hudson AG, Jiggins C, Jones J, Keller B, Marczewski T, Mallet J, Martinez-Rodriguez P, Möst M, Mullen S, Nichols R, Nolte AW, Parisod C, Pfennig K, Rice AM, Ritchie MG, Seifert B, Smadja CM, Stelkens R, Szymura JM, Väinölä R, Wolf JBW, Zinner D (2013) Hybridization and speciation. Journal of Evolutionary Biology, 26, 229-246.

Darwin C (1929) The origin of species by means of natural selection. American Anthropologist, 61, 176-177. 
Frank SA (2011) Natural selection. II. Developmental variability and evolutionary rate. Journal of Evolutionary Biology, 24, 2310-2320.

Hedberg O (1958) The taxonomic treatment of vicarious taxa. Uppsala Universitets Arsskrift, 6, 186-195.

Hong DY (2016) Biodiversity pursuits need a scientific and operative species concept. Biodiversity Science, 24, 979-999. (in Chinese with English abstract) [洪德元 (2016) 生物多样性事业需要科学、可操作的物种概念. 生物多样 性, 24, 979-999.]

Li QJ, Li Y (2010) Lamarck redux-A revisit of Darwinism. Journal of Biology, 27(2), 55-57. (in Chinese with English abstract) [李启剑, 李越 (2010) 拉马克的归来: 对达尔文 主义的再审视. 生物学杂志, 27(2), 55-57.]

Liu JQ (2016) “The integrative species concept” and "species on the speciation way”. Biodiversity Science, 24, 1004-1008. (in Chinese with English abstract) [刘建全 (2016) “整合物种概念” 和 “分化路上的物种”. 生物多样 性, 24, 1004-1008.]

Lu BR, Wang Z (2016) What is a species: Conflict between evolutionary continuity and taxonomic discontinuity. Chinese Science Bulletin, 61, 26632669. (in Chinese with English abstract) [卢宝荣, 王哲 (2016) 什么是物种: 进
化连续性与分类间断性冲突的产物. 科学通报, 61, 2663-2669.]

Maynard Smith J (1982) Evolution and the theory of games. Cambridge University Press, Cambridge.

Traulsen A, Iwasa Y, Nowak MA (2007) The fastest evolutionary trajectory. Journal of Theoretical Biology, 249, 617-623.

Nowak MA (2006) Evolutionary Dynamics: Exploring the Equations of Life. Harvard University Press, Cambridge.

Wang RW (2021) The End of Rationality and Selfness-A Story on the Asymmetry, Uncertainty and Evolution of Cooperation. China Commerce and Trade Press, Beijing. (in Chinese) (in press) [王瑞武 (2021) 理性与自私的终结 一非对称性、不确定性与社会合作行为. 中国商务出版 社, 北京.]

Wilkins JS (2009) Species: A History of the Idea. University of California Press, Berkeley.

Zhou CF, Yang G (2011) Existence and Definition of Species. Science Press, Beijing. (in Chinese) [周长发, 杨光 (2011) 物种的存在与定义. 科学出版社, 北京.]

(责任编委: 王志恒 责任编辑: 周玉荣)

\section{附录 Supplementary Material}

\section{附录1 有关实例验证的说明}

Appendix 1 Notes on no case is given to verify the defination http://www.biodiversity-science.net/fileup/PDF/2020276-1.pdf 
李敏岗，王超，王瑞武 (2021) 路径依赖下的物种形成机制. 生物多样性, 29(3), 409-418.

http://www.biodiversity-science.net/CN/10.17520/biods.2020276

\section{附录1 有关实例验证的说明}

Appendix 1 Notes on no case is given to verified the defination

首先，本文中我们旨在理论层面给出一种关于物种形成的机制解释，以及在该机制下的物种分类方法， 供大家认识和讨论。然而从理论到实际应用还需要根据真实的系统考虑很多问题, 我们正在寻找合适的实 验生物学家，讨论如何合作开展对这一理论的验证。

其次, 由于理论与实际应用之间存在很大的差距，一个理论在发展初期也许并不能通过实验被很好地 验证, 而是需要后续的很多工作进一步完善才能体现出它的优劣性。譬如, Kimura在1953年通过建立数学模 型得到了“分子层面的中性选择”, 并没有给予任何实例(Kimura, 1953), 但正是这一数学理论模型的提出, 中性理论才得以发展起来, 并成为生物学中最重要的思想之一。在提出初期同样未能得到验证的还有 Hamilton提出的“亲选择”理论(Hamilton,1963; Queller, 1992), 该理论后来发展成为自达尔文以来最伟大的 进化理论。反过来, 在实际物种分类中被主要应用的支序分类法, 当时也只是一种经验性的操作, 并没有理 论上关于其种群遗传学或者分类系统学严格的逻辑证明。

另外，限于作者从事理论生态研究的背景，我们目前还没有足够的生物学和分类学背景去进行实际操 作, 以验证该理论的合理性。这方面的工作需要很多从事分类工作的科学家共同完成, 这也是我们未来工 作很重要的一部分。

最后, 实际上关于物种进化是依赖路径的这一想法, 四川大学教授刘建全也已经意识到并在其文章中 提到(刘建全, 2016)。我们在文章中阐述了路径依赖的内在机制，并通过模拟手段对依赖路径形成物种这一 过程进行了可视化展示。而关于物种的分类方法的确定，我们是借鉴了高能物理和核物理实验中用来鉴别 不同粒子的方法。实验上观测粒子衰变过程观测到的能谱通常是多个粒子叠加起来的高高低低的峰, 实验 用半高宽来鉴别不同的粒子种类。目前这一统计方法已经被广泛应用于高能物理和核物理实验，是粒子种 类鉴别和粒子分类的主要方法。我们将这一统计方法推广到物种的分类是有可行性的。

\section{参考文献}

Kimura M (1954) Process leading to quasi-fixation of genes in natural populations due to random fluctuation of selection intensities. Genetics, 39, 280-295.

Hamilton WD (1963) The evolution of altruistic behavior. The American Naturalist, 97, 354-356.

Queller DC (1992) A general model for kin selection. Evolution, 46, 376-380.

Liu JQ (2016) "The integrative species concept" and "species on the speciation way". Biodiversity Science, 24, 1004-1008. (in Chinese with English abstract) [刘建全 (2016) “整合物种概念”和“分化路上的物种”. 生物多样性, 24, 1004-1008.] 